\title{
Using PsyScope for demonstrations and student-designed experiments in cognitive psychology courses
}

\author{
JONATHAN VAUGHAN and PENNY L. YEE \\ Hamilton College, Clinton, New York
}

\begin{abstract}
We describe a plan for integrating an experimental control language, PsyScope, into undergraduate laboratory exercises of perceptual and cognitive experiments on Macintosh computers. PsyScope is a powerful and versatile system with which students can modify standard research paradigms and execute experiments of their own design, thus facilitating student-initiated independent research. Data are summarized with a general-purpose program, PsySquash, for import into Statview or SuperAnova for further analysis. This system provides an effective means of implementing student projects.
\end{abstract}

How do you provide an enriching laboratory experience for undergraduate psychology students who have minimal technical knowledge about computers? This problem is faced by many educators who wish to introduce students to empirical research by providing a rich laboratory experience. A primary goal of our courses in cognitive psychology and in attention and performance is to provide students with hands-on experience in the design of new experiments in addition to data collection and analysis. Students best understand the scientific process by researching and testing their own ideas with real empirical data. However, a major dilemma for undergraduate institutions is that few students possess enough computer expertise to test questions that are of interest to them. Our experience suggests that the typical student has no more than a working knowledge of the standard Macintosh interface and some word-processing skills.

A common approach to this problem has been the development of software packages to facilitate the program development of computerized experiments, using either special-purpose tachistoscope programs (Barron, 1992; Bharucha, Meike, \& Baird, 1987; Busey, 1992; Doenias, Langland, \& Reisberg, 1992; Enns \& Rensink, 1991), or more general experimental control programs for the

\footnotetext{
We are grateful to Jonathan Cohen and Brian MacWhinney for the development of the PsyScope system and for permission to use examples from it, to Earl Hunt, Simon Farr, Colene McKee, and John Palmer for allowing us to incorporate the Squash statistical routines into PsySquash, and to Helene Joseph for beta testing of PsySquash and preparation of documentation. The development of Psyscope has been made possible by the contributions of the PsyScope Consortium. Inquiries about PsyScope should be directed to Jonathan Cohen (jc5e+@andrew.cmu.edu) or Brian MacWhinney (brian+@andrew.cmu.edu). Inquiries about PsySquash and its tutorial materials should be directed to J. Vaughan or P. Yee, Department of Psychology, Hamilton College, 198 College Hill Road, Clinton, NY 13323 (e-mail: jvaughan@hamilton.edu or pyee@hamilton.edu). PsySquash is made available at no charge for noncommercial, educational use, and it will be posted on the MacPsych archive, which is accessible by anonymous FTP at macpsych@stolaf.edu.
}

Macintosh (Chute, 1986; Haxby, Parasuraman, Lalonde, \& Abboud, 1993) or IBM compatibles (Schneider, 1989). (See Goolkasian, 1993, for a review of these and other instructional programs for the Macintosh.)

The advantages of these packages are that little or no programming experience is necessary to implement a study. However, in exchange for simple implementation, the complexity of the designs that are easily accommodated by the software is often limited. For example, to the authors' knowledge, none of the packages mentioned above provide for the automatic randomization of trial sequences or the automatic orthogonal combination of different independent variables within an experiment.

At Hamilton, we have adopted a solution that we believe provides the simplicity necessary for second- or third-year undergraduates to tackle relatively complex experimental designs while retaining access to the power often needed for the more advanced researcher. The experiment-design package we have adopted for our laboratories is PsyScope.

\section{PSYSCOPE}

PsyScope, developed by Cohen, MacWhinney, Flatt, and Provost (1993), is a powerful and versatile experimental control system for Macintosh computers. PsyScope represents experimental paradigms in two ways simultaneously: iconically, in a graphic user interface (GUI), and textually, in a directly editable script. The user's changes in the iconic representation are immediately reflected in the script, and (insofar as possible) vice versa. PsyScope incorporates many sophisticated features that are useful in the research laboratory. For example, it affords the automatic randomization and factorialization of the stimuli presented in an experiment, as well as the modification of stimulus attributes (color, position, font, sound, etc.), as required by the paradigm. In addition, because the experimental design is accessible in an editable script 
with full programming capabilities, more complex needs can be accommodated, such as providing customized performance feedback after each block of trials and repeating trials on which errors have occurred.

Although PsyScope has many powerful design features, we find that its basic capabilities suffice for most undergraduate projects. Consequently, in our courses we provide general instruction on only a subset of PsyScope's features. We have adopted this approach for two reasons. The first is pedagogical: a primary goal in our laboratory courses is to give students practical experience with research applications and experimental design. For example, we have found it valuable to require students to work out the counterbalancing of their experimental designs by hand, even though the counterbalancing of factorial designs could be delegated to PsyScope. The second reason is pragmatic: most student applications do not require all of the power that the PsyScope system can provide. Restricting students to only a subset of PsyScope's features-in particular, a subset that does not require them to learn any new computer techniques-reduces the startup time for students in the lab. Our students are ready to implement a wide range of research designs after a relatively small investment in teaching some basic features of PsyScope. With this approach, we can give students access to a system where they can learn about experimental design and data acquisition while having to invest only minimal time in learning the specifics of computer programming. Finally, students who need to exploit the full power of PsyScope (e.g., for an ambitious senior project) can do so most efficiently after having worked with the package in our formal lab courses.

In this paper we describe two aspects of our work with students in intermediate and advanced laboratory courses in experimental psychology. The first is how students are given expertise in experimental design by using the PsyScope system. The second is how students can use a special-purpose program, PsySquash, to prepare the resulting data for analysis by a commercial statistical package.

\section{EXPERIMENTAL DESIGN}

We move students through a five-step process when developing new experiments with PsyScope and PsySquash. Once a research question has been identified, each student first works out the details of his/her design by determining the appropriate conditions, stimulus materials, and counterbalancing techniques to be used. Second, the student translates the design and stimulus information into a text file. Each line in the file corresponds to a different trial type, where all information that distinguishes one trial from another is specified, such as the condition label, the stimulus, and the correct response. The third step requires the student to link this file to the Trial Template in PsyScope. The student then conducts the experiment. In the final step, the student selects appropriate summary statistics and breakdowns of the data. The breakdown of data by experimental conditions and subjects is immediately available for import into the Statview or SuperAnova statistical packages (Abacus Concepts, Inc., Berkeley, CA).

\section{Basic PsyScope Features}

We provide below a brief description of the main features we have exploited in implementing PsyScope with our students, followed by a detailed description of an example script. For information about PsyScope's other capabilities, see Cohen et al.'s original (1993) publication.

Within the GUI view of PsyScope, an experiment is represented as several iconic objects that are linked on a desktop, with the experiment's name at the highest node (see Cohen et al., 1993, Figure 13). Beneath the experiment object icon are major components of the experiment, called blocks. The experimenter specifies, within the experiment object icon, how many times to access each block and the order in which to run the component parts (randomly, sequentially, etc.). We typically use one block to present instructions to the subject and a second block to present trials. Linked to each block is a trial template, which defines the events to be presented in that block of trials. (See Figure 5 in Cohen et al., 1993, for an illustration of the possible events and a schematic of a trial template.) Within the icon for a block, the experimenter specifies how many times in the experiment to run the trial template.

A key feature in the design window that we rely on is the external list file. This object accesses the text file prepared by the student, and parses the information into appropriate fields that ultimately define a trial. For example, the list icon will sort information about the condition, the stimulus, and the correct response for each trial. The trial template object then retrieves the sorted information from the list icon to fill in the details of the changing values for different trials.

We have used PsyScope to conduct some 20 different experiments, ranging from replications of the "classics" to entirely novel student-designed experiments. Here, we will describe one example in detail-PsyScope's imple-

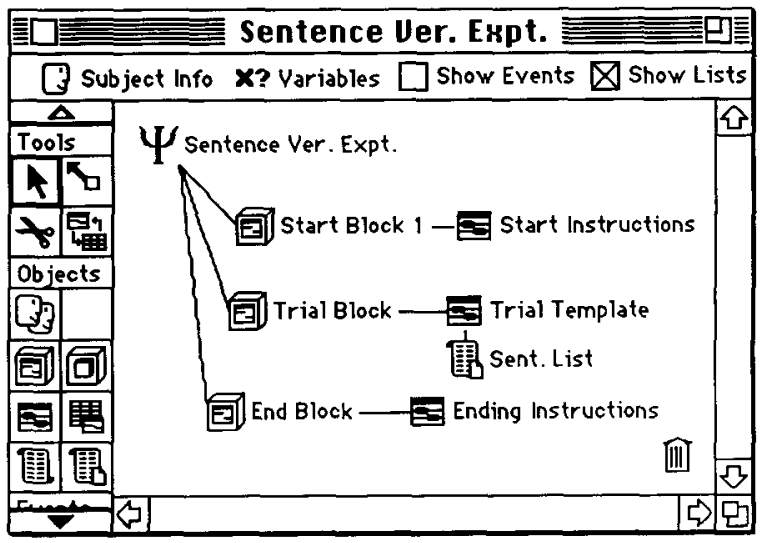

Figure 1. PsyScope's graphic user interface (GUI) representation of the sentence-picture verification experiment. 


\begin{tabular}{l}
\hline Tools $\nabla[:$ Edit File \\
\hline Load as Script
\end{tabular}

Figure 2. Frontmost window: A sample trial from the sentence-picture verification experiment. Rearmost window: External text file which specifies trial types and design for the sentence-picture verification experiment.

mentation of Clark and Chase's (1972) sentence-picture verification paradigm. We have selected this example because most readers will be familiar with the underlying research, and because it illustrates several aspects of the use of PsyScope.

\section{Example: Implementing the Sentence-Picture Verification Paradigm in PsyScope}

Figure 1 presents the general structure of the experiment's script, as represented in PsyScope's GUI. In this lab exercise, subjects are asked to compare a simple sentence description with a picture and report the accuracy of the statement by pressing one of two keys. The frontmost window of Figure 2 illustrates a sample trial from this experiment. Some sentences are in simple declarative form, such as "The star is above the plus," whereas others are more complex- "The star is not below the plus." The sentences vary across three different independent variables. The sentence can (1) be a true or false description of the picture, (2) be expressed in a declarative or negative form, and (3) use an unmarked ("above") or marked ("below") form of the adverb describing the relative position of the two symbols. When each of these sentences is paired with two symbol relations (plus above star and star above plus), 16 unique trial types constitute a balanced experimental design.

\section{The External Text File}

Once the specifics of the experimental trials are determined, the next step is to translate this information into a text file. The rearmost window of Figure 2 shows a text file named "Sent Stim," which specifies information about each of the 16 trial types (of which 7 are shown here). Each line in the text file corresponds to a single trial type. By convention, we use the first column in the text file to specify the condition label. When there is more than one independent-variable factor, the levels are separated by the underscore character. Thus, in the sentencepicture verification paradigm, all trials that are true, declarative, and unmarked are labeled 'T_D_UM' in the first column. Subsequent columns in the text file spec- ify, for each trial, the symbol to be presented at the top of the picture (the star or the plus), the symbol to be presented at the bottom (the plus or the star), the corresponding sentence, which key is correct on that trial, and which key is an error.

\section{The External List Object in PsyScope}

Once the text file is complete, PsyScope needs to interpret the contents of the file. This is accomplished with the external list object, in this case named "Sent. List." Figure 3 presents the window for the external list object. The "Set..." button in the second line of the window allows the student to specify the text file to be read by PsyScope. When it is selected, PsyScope presents a menu of files from which the user selects one to be linked with the object. In this example, the stimulus text file has been

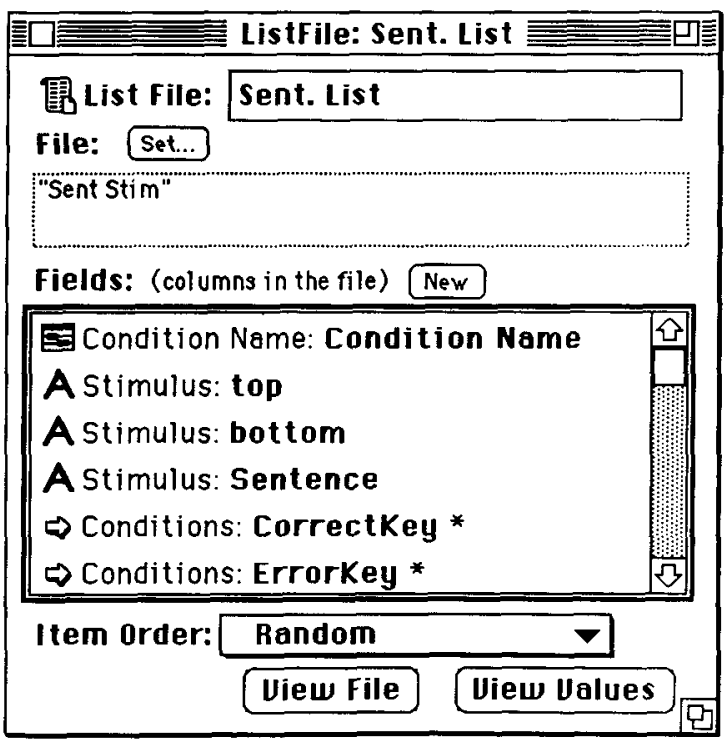

Figure 3. The window for the External List Object, which specifies the contents of each column of an external list text file (see Figure 2). 


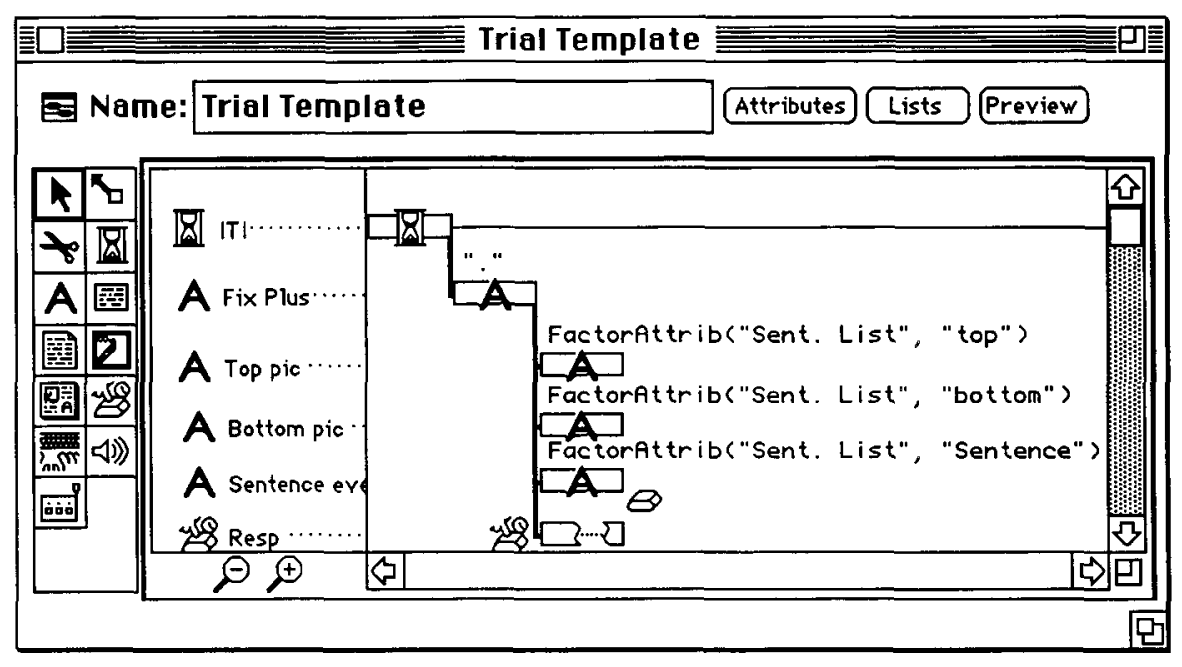

Figure 4. Trial Template for the sentence-picture verification experiment.

set to a file named "Sent Stim." Once the file is linked, the user defines the type of information provided in each of the columns (fields) of the text file (e.g., information about the condition type, stimulus type, or correct and incorrect responses). The first field defined corresponds to the first column of information in the text file, the second field corresponds to the second column of information, and so on.

\section{The Trial Template: Event Attributes That Are Constant}

The next step is to define the events that are common to each trial by using the trial template object. For the sentence-picture verification experiment, there are six events in each trial: an intertrial interval (ITI), a fixation point (Fix Plus), the symbol to appear on the top (Top pic), the symbol to appear on the bottom (Bottom pic), the sentence (Sentence), and an event to record the subject's response (Resp). The events in a trial template are graphically presented on a time line (moving from left to right) that shows the onset and offset of each event (Figure 4).

Each event's attributes are set by selecting the event. Highlighting an event causes the temporal attributes of the event to appear at the bottom of the template window, so that the times of onset and offset of the stimulus event can be changed. Other features, such as font, size, style, color, and position, can easily be set (see Figure 7 in Cohen et al., 1993).

\section{The Trial Template: Event Attributes That Vary}

Some attributes of a stimulus event may have to change on each trial, such as the sentence and the picture in the sentence-verification experiment. Varying attributes are handled by changing the setting for "Stimulus" in the attributes window. The values for "Sentence Event" stimuli are found in the external list file, so the setting for the stimulus (top attribute) is changed to indicate that the stimulus will "Vary by" a "List."

The last event to define in the trial template is the response event, which records information about the subject's response on each trial. Here, the user defines the correct and incorrect response and specifies the information to be stored in the data file (in this case, reaction times and accuracy).

When all events have been appropriately defined, a sample trial can be previewed by clicking in the preview box of the trial template. When the experiment is run, separate data files are stored for each subject (see Figure 5). These can then be read by the PsySquash analysis program (see below) to generate summary statistics.

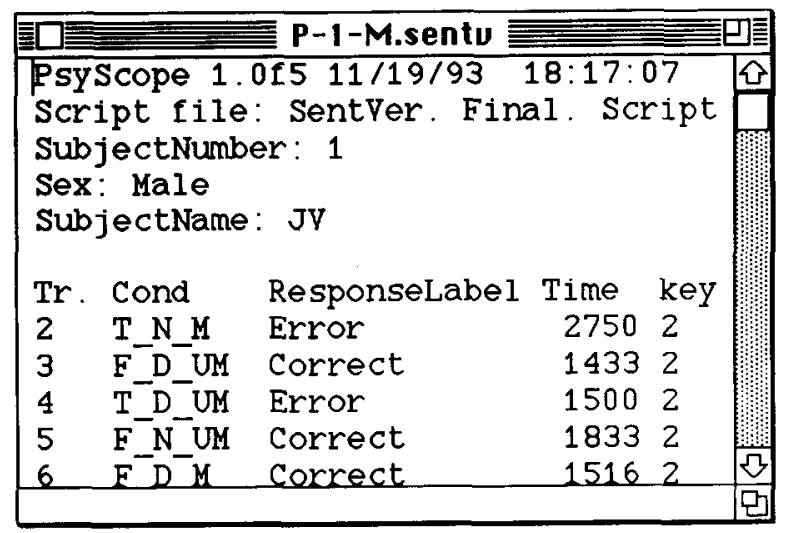

Figure 5. Typical PsyScope data file from the sentence-picture verification experiment. The "Cond" (condition) column indicates whether the test sentence was true or false, declarative or negative, and marked or unmarked. The "ResponseLabel," "Time," and "Key" columns indicate the correctness, reaction time, and response of the subject on each trial. 


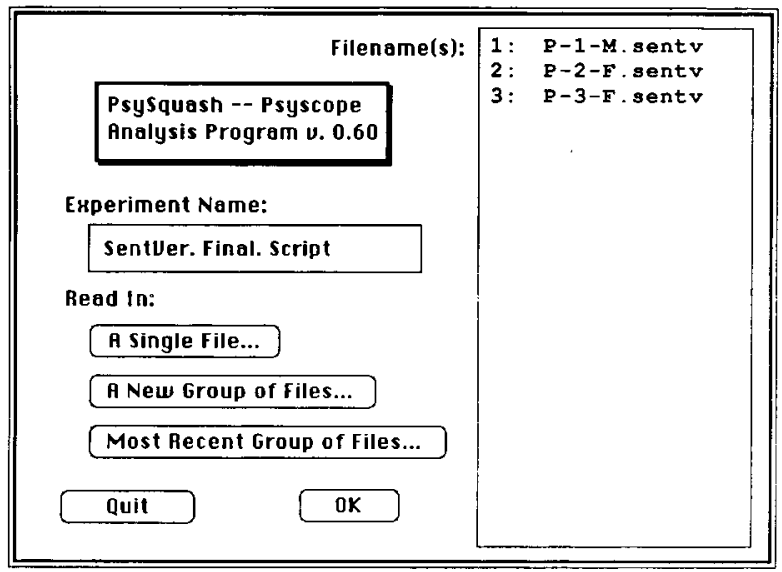

Figure 6. File selection window of PsySquash. The group of files that has been selected is remembered so that similar analyses can be performed on the same data.

\section{Advantages of the PsyScope Implementation}

Many standard paradigms can be modified by altering the stimulus list or the attributes of the trial template. We explain to the student how the stimulus list must be constructed, and then have the student construct the stimulus item list (words, stimulus attributes, and trial labels) by using a word processor with which he/she is already familiar. This permits the stimulus generation to be done on a student's own computer or at the campus computer center, so it is not necessary for the students to have individual copies of PsyScope in order to work on their projects. At the next laboratory meeting, it is then a simple matter for the instructor or laboratory assistant to demonstrate how to link the student's trial list to an experiment prototype so that the appropriate trials will be presented.

Because students generate their stimulus lists outside the PsyScope environment, they are not able to take advantage of all of its sophisticated design tools. However, we wish to impose on the students (who are just learning the principles of good experimental design) complete responsibility for generating their own stimulus lists, including, for example, all appropriate combinations of stimuli for their factorial design, such as counterbalancing stimulus types. This approach allows class time to be reserved for issues related to the theoretical background of the students' projects and on issues of experimental design, while minimizing what the students must learn about the inner workings of PsyScope.

\section{PREPARING DATA FOR ANALYSIS BY USING PSYSQUASH}

Data analysis proceeds in two phases outside of the PsyScope environment. In the first phase, the raw data are sorted and summary statistics are computed for requested conditions by using PsySquash, a Pascal program written for this purpose. In the second phase, the data are analyzed by using a commercial statistical package. The mechanics of running PsySquash are straightforward, given knowledge of the variables present in the study. PsySquash is designed to lead students through the analysis by using constructs that are familiar from their class work in statistics.

PsySquash first prompts the user to specify one or several PsyScope data files for analysis (see Figure 6). The program scans each column of the input data to enumerate

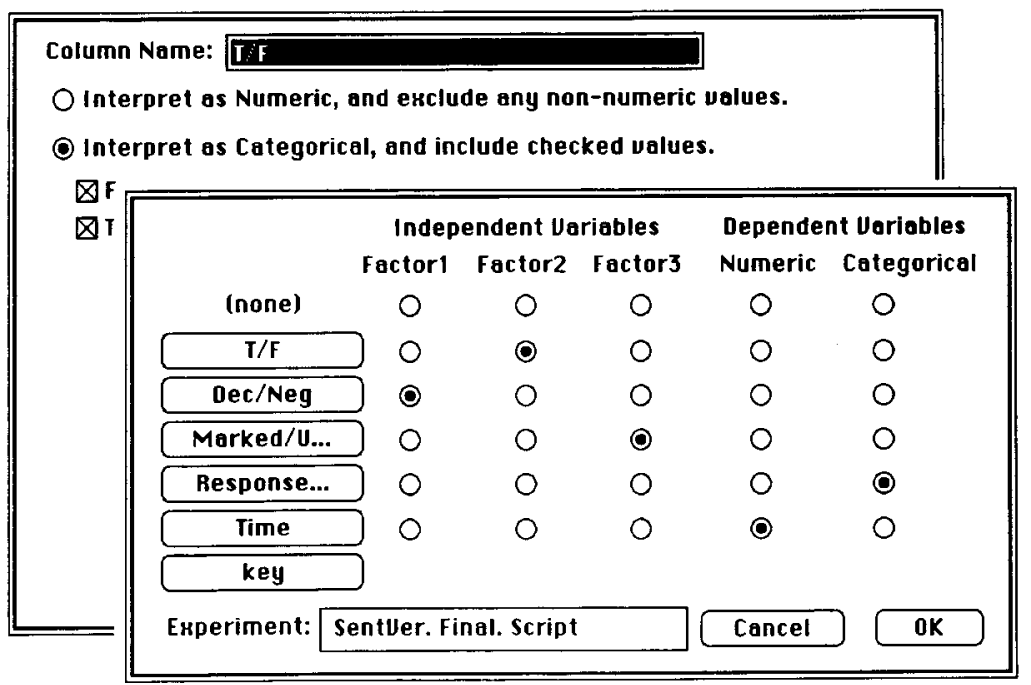

Figure 7. Rearmost window: Naming of a variable, and specification of its interpretation by PsySquash as either numeric or categorical. Frontmost window: Selection of independent and dependent variables in PsySquash. Up to nine columns of the data file can be displayed. Note that the order of selection of the independent-variable factors-Factor1, Factor2, and Factor3-will determine how these factors are nested in the analysis summary. 


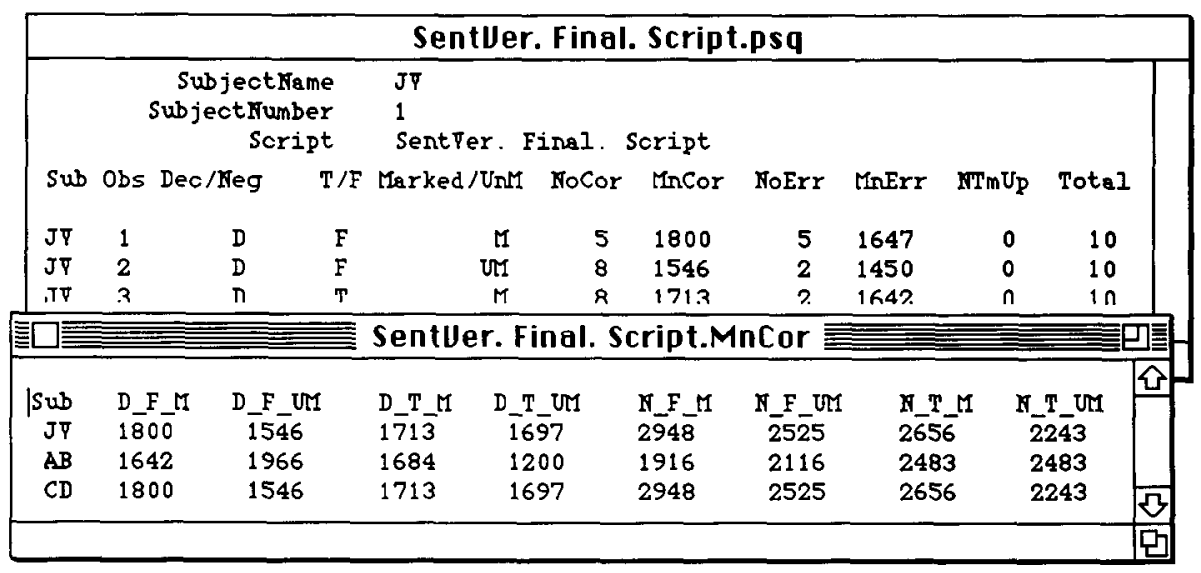

Figure 8. Rearmost window: PsySquash data summary for 1 subject. Frontmost window: Data for 3 subjects, formatted by PsySquash for import into Statview or SuperAnova for repeated measures analysis. Note that independent variables are nested in the order specified by the variable selection in Figure 4.

the values that appear in it. (The "Conditions" column is automatically expanded so that each independent variable is separately represented.) Next, it is the student's responsibility to specify which columns represent levels of an independent-variable factor (up to three factors, each with up to 10 levels), and which represent the dependent variables, which may be either categorical or numeric (Figure 7, rearmost window). (The experiment may comprise more than three independent variables. We find in our courses that three factors suffice for most class exercises. If there are more than three factors, the data may be examined with a different set of selected independent variables.) To facilitate the specification of the independent and dependent variables, and for heuristic purposes, PsySquash displays, for each column, all the values found in that column (Figure 7 , frontmost window). Seeing the values that are found in the column helps the student recognize that column as belonging to an independent or a dependent variable, and as representing a numeric or categorical scale of measurement. In multifactor designs, it is desirable to be able to manipulate how the different independent-variable factors are nested in the eventual output. The variable selection allows the student to select not only which independent variables to use in the analysis, but also how they are to be nested in the output data, depending on which columns are assigned to Factor 1 , Factor 2 , and Factor 3. In the resulting data summary, Factor 3 is nested within Factor2, which in turn is nested within Factor1. In the example, the Marked/Unmarked factor is nested within $\mathrm{T} / \mathrm{F}$, which in turn is nested within the Dec/Neg sentence type factor. Finally, the student selects which dependent measures are to be computed from the data set, selecting the desired measures of frequency, central tendency, and dispersion of correct and incorrect responses. For convenience, a default set of descriptive statistics may be selected (frequency and mean of correct, error, and time-out responses). The data are summarized in an output file by experimental condition (Figure 8, rearmost window) and, if appropriate, may be saved in a format suitable for import into Statview or SuperAnova (Figure 8 , frontmost window).

\section{SUMMARY}

The combination of PsyScope and PsySquash provides an effective enhancement of the laboratory experience for undergraduate psychology students. A workbook of tutorial exercises to assist students in learning to use PsyScope is currently under development and is available from the authors.

\section{REFERENCES}

BArron, C. (1992). Tachistoscopic software for the Macintosh. Behavior Research Methods, Instruments, \& Computers, 24, 431-433.

Bharucha, J. J., Meike, B., \& Baird, J. C. (1987). The Macintosh as a user-friendly laboratory for perception and cognition. Behavior Research Methods, Instruments, \& Computers, 19, 131-134.

BUSEY, T. (1992). Tachistoscopic software for Macintosh computers. Behavior Research Methods, Instruments, \& Computers, 24, 426-430.

Chute, D. L. (1986). MacLaboratory for psychology: General experimental psychology with Apple's Macintosh. Behavior Research Methods, Instruments, \& Computers, 18, 205-209.

Clark, H. H., \& Chase, W. G. (1972). On the process of comparing sentences against pictures. Cognitive Psychology, 3, 472-517.

Cohen, J., MacWhinney, B., Flatt, M., \& Provost, J. (1993). PsyScope: An interactive graphic system for designing and controlling experiments in the psychology laboratory using Macintosh computers. Behavior Research Methods, Instruments, \& Computers, 25, 257-271.

Doenias, J. M., Langland, S. E., \& Reisberg, D. (1992). A versatile, user-friendly tachistoscope for the Macintosh. Behavior Research Methods, Instruments, \& Computers, 24, 434-438.

ENNS, J. T., \& RENSINK, R. A. (1991). VSearch Color: Full-color visual search experiments on the Macintosh II. Behavior Research Methods, Instruments, \& Computers, 23, 265-272.

GOOLKASIAN, P. (1993). A review of Macintosh-based instructional software. Behavior Research Methods, Instruments, \& Computers, 25 , 164-167.

Haxby, J. V., Parasuraman, R., Lalonde, F., \& Abboud, H. (1993). SuperLab: General-purpose Macintosh software for human experimental psychology and psychological testing. Behavior Research Methods, Instruments, \& Computers, 25, 400-405.

SCHNEIDER, W. (1989). Enhancing a standard experimental delivery system (MEL) for advanced psychological experimentation. Behavior Research Methods, Instruments, \& Computers, 21, 240-244. 